\title{
Analytic Study of the Migdal-Kadanoff Recursion Formula
}

\author{
K. R. Ito* \\ ZiF, Bielefeld University, Wellenberg 1, D-4800 Bielefeld 1, Federal Republic of Germany
}

\begin{abstract}
After proposing lattice gauge field models in which the Migdal renormalization group recursion formulas are exact, we study the recursion formulas analytically. If $D$ is less than 4 , it is shown that the effective actions of $D$-dimensional $U(1)$ lattice gauge models are uniformly driven to the high temperature region no matter how low the initial temperature is. If the initial temperature is large enough, this holds for any $D$ and gauge group $G$. These are also the cases for the recursion formulas of Kadanoff type. It turns out, however, that the string tension for $D=3$ obtained by these methods is rather big compared with the one already obtained by Mack, Göpfert and by the present author. The reason is clarified.
\end{abstract}

\section{Introduction}

A decade ago, Migdal [1] proposed renormalization group recursion formulas which are rather simple but are believed to approximate the real systems fairly well. After his works, Kadanoff [2] reconstructed similar recursion formulas by his decimation methods. The most surprising feature of these recursion formulas is that the recursion formulas of $D$-dimensional lattice gauge models are equivalent to those of $(D / 2)$-dimensional statistical mechanical models (with nearest neighbor interactions). One advantage is that these recursion formulas have a closed form and then they may be solved analytically.

But it remains to see to what extent these approximate formulas are close to the real systems. We answer this question by solving the recursion formulas analytically. The answer is quite negative at least when $D$ is less than 4 : the effective dimensions are too close to two if $D<4$. Thus these methods give larger string tensions compared with the real ones.

Before writing down these formulas, we propose special lattice gauge field models in which the recursion formulas of these types are exact. This may help the

* On leave of absence from: Dept. of Maths. Bedford College, Regents Park, London NW 1, England

Address after 1.10.1984: Dept. of Maths. College of Liberal Arts, Kyoto University, Sakyoku, Kyoto 606, Japan 


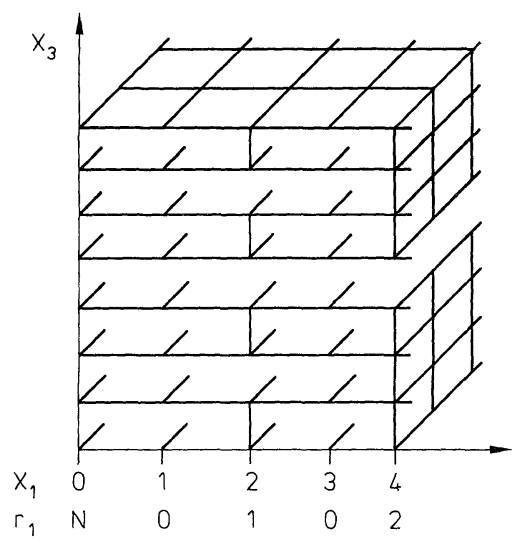

Fig. 1. Realization of the construction of the model in which the Migdal recursion formula is exact. Actions for the plaquettes on the vertical planes are zero or infinite

reader to see what may be wrong in these formulas. This idea was borrowed from [3]. We first consider a 3-dimensional case setting a rectangular box of size $\lambda^{N} \times \lambda^{N}$ $\times\left(\lambda^{N}-1\right)$ in $Z^{3}$ with its corners at $\left(T_{1}, T_{2}, T_{3}\right), T_{i}=0$ or $\lambda^{N}\left(\lambda^{N}-1\right.$ if $\left.i=3\right)$. Here $\lambda$ is an integer such that $\geqq 2$. Let $G$ be a compact group and let $U(G)$ be one of its representations. Let $\Xi$ be our rectangular box.

(i) Choose the temporal gauge. Namely set $G \ni v_{b}=1$ for the vertical bonds $b=\left(x, x+e_{3}\right) \in \Xi$.

(ii) For each plaquette (unit square) $p$ on $x_{1}-x_{2}$ planes in $\Xi$, there corresponds the standard Wilson-type action:

$$
p \rightarrow A_{p}=\beta \operatorname{Re} \operatorname{Tr} U\left(v_{p}\right), \quad v_{p}=\prod_{b \in \partial p} v_{b},
$$

where $\beta=\left(2 g_{0}^{2}\right)^{-1}$ is the (initial) inverse temperature.

(iii) The actions for the plaquettes on $x_{1}-x_{3}$ and $x_{2}-x_{3}$ planes (vertical planes) in $\Xi$ are zero $(\beta=0)$ or ferromagnetically infinite $(\beta=\infty)$, depending on where they are. For example, $x_{2}-x_{3}$ planes are indexed by $x_{1}=0,1, \ldots, \lambda^{N}$. Let $x_{1}$ $=p_{1} \lambda^{r_{1}}+p_{2} \lambda^{r_{2}}+\ldots$, where $p_{i}=1,2, \ldots, \lambda-1$ and $r_{1}<r_{2}<\ldots$ (positive integers). Then the $x_{2}-x_{3}$ plane at $x_{1}$ is partitioned into non-overlapping parallel rectangles of equal sizes $\lambda^{N} \times\left(\lambda^{r_{1}}-1\right)$. They are separated by a distance one in the $x_{3^{-}}$ direction. There correspond the ferromagnetically infinite actions $(\beta=\infty)$ to the plaquettes in each of these rectangles. In other words, $v_{b}$ and $v_{b^{\prime}}$ are set equal when $b=\left(x, x+e_{2}\right)$, and $b^{\prime}=\left(x^{\prime}, x^{\prime}+e_{2}\right)$ are in each of these rectangles and nearest neighbors to each other. $\beta$ is set at zero for other plaquettes in this $x_{2}-x_{3}$ plane which are not contained in these rectangles. $\left(x_{1}=0\right.$ is regarded as $\lambda^{N}$.)

(iv) Repeat (iii) for the plaquettes on the $x_{1}-x_{3}$ planes in $\Xi$.

This method is easily extended to higher dimensions, which is left to the reader. Our construction may be well understood by Figs. 1 and 2 which correspond to $D=3$ and $\lambda=2$. Note that $\beta$ is set at zero for vertical plaquettes if $r_{1}=0$ (i.e. $x_{1}\left(x_{2}\right)$ $=$ odd in this case.)

Let $g^{(0)}\left(v_{p}\right)=\exp \left[\beta \operatorname{Re} \operatorname{Tr} U\left(v_{p}\right)\right]$. Then this system obviously satisfies the following recursion formulas of Migdal: 


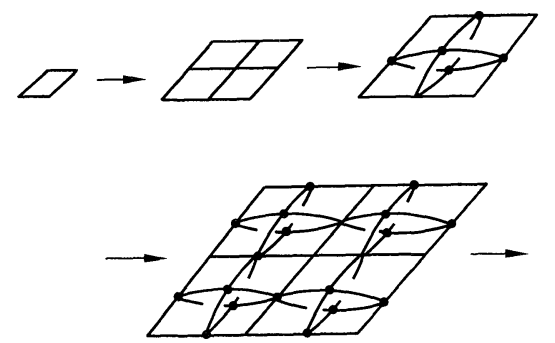

Fig. 2. Iteration of the recursion formulas for this system. This corresponds to $D=3$ and $\lambda=2$. Two rectangles, each containing 4 plaquettes, are combined with each other with their boundaries identified. Internal bonds are independent

$$
g^{(n)}(v)=(\mathscr{N})^{-1}\left[\int \prod_{p \subset \Lambda} g^{(n-1)}\left(v_{p}\right) \prod_{b \in \Lambda^{0}} d v_{b}\right]^{\lambda s},
$$

where $\Lambda$ is a rectangle of size $\lambda \times \lambda$ (in the unit of $\lambda^{n-1}$ ) containing $\lambda^{2}$ plaquettes in it, $b \in \Lambda^{0}$ bonds which are inside of $\Lambda, d v_{b}$ the Haar measure, $s=D-2$ and $v=\prod_{b \in \partial \partial} v_{b}$, where the product is taken following the direction of the boundary of $\Lambda . \lambda^{s}$ is the number of the rectangles of size $\lambda \times \lambda$ to be gathered. By suitable gauge transformations, we have

$$
\begin{aligned}
g^{(n)}(v)= & (\mathscr{N})^{-1}\left[\int g^{(n-1)}\left(v v_{1}^{-1}\right) g^{(n-1)}\left(v_{1} v_{2}^{-1}\right) \ldots\right. \\
& \left.\times g^{(n-1)}\left(v_{\lambda^{2}-2} v_{\lambda^{2}-1}^{-1}\right) g^{(n-1)}\left(v_{\lambda^{2}-1}\right) \prod d v_{i}\right]^{\lambda s} .
\end{aligned}
$$

Here $\mathcal{N}$ is chosen so that $g(v=1)=1$. This is called the recursion formula of Migdal type (M type for short).

By replacing $\lambda^{N} \times\left(\lambda^{r_{1}}-1\right)$ by $\lambda^{N} \times\left(\lambda^{r_{1}+1}-1\right)$ in step (iii) in the previous construction, we obtain the lattice gauge model that satisfies the Kadanoff recursion formulas exactly $\left(x_{1}=0\right.$ and $x_{1}=\lambda^{N}$ are regarded as $\left.\lambda^{N-1}\right)$ :

$$
g^{(n)}(v)=(\mathscr{N})^{-1} \int\left[g^{(n-1)}\left(v v_{1}^{-1}\right) \ldots g^{(n-1)}\left(v_{\lambda^{2}-1}\right)\right]^{\lambda s} \Pi d v_{i} .
$$

This recursion formula (called the Kadanoff type) was originally obtained by Kadanoff by his potential moving methods. It is recommended to draw figures that correspond to Figs. 1 and 2. It is easily seen that more vertical plaquettes have $\beta=\infty$ couplings in this system.

These are equivalent to the renormalization group recursion formulas of statistical mechanical models with nearest neighbor interactions. In this case $v_{i}$ is replaced by $s_{i} \in S^{\ell}\left(\ell\right.$ dimensional sphere for example), $v v_{1}^{-1}$ by $s s_{1}, v_{i} v_{i+1}^{-1}$ by $s_{i} s_{i+1}$, $v_{\lambda^{2}-1}$ by $s_{\lambda^{2}-1} \cdot s^{\prime}$ and finally $g^{(n)}(v)$ is replaced by $g^{(n)}\left(s s^{\prime}\right)$. Moreover $\lambda^{s}$ becomes $\lambda^{2(D-1)}$ in this case.

These recursion formulas may look quite simple, but it is rather hard to investigate these analytically except for the case of $s=0$. There have been some computer experiments, see $[4,5]$. See also [6], but unfortunately the details of $[6]$ remain to be seen. 
In the case of the $D$ dimensional $U(1)$ lattice gauge model or in the case of the $(D / 2)$ dimensional XY-type model, we replace $v$ by $\theta \in[-\pi, \pi), v_{i}$ by $\theta_{i} \in[-\pi, \pi)$ and $v_{i} v_{i+1}^{-1}$ is replaced by $\theta_{i}-\theta_{i+1}$. Then it is explicit that these two different models have the same recursion formulas. In this case we start with the Villain action,

$$
g^{(0)}(\theta)=\text { const } \sum_{n} \exp \left[-\frac{\beta}{2}(\theta+2 n \pi)^{2}\right],
$$

or with the action of the $X Y$ model type:

$$
g^{(0)}(\theta)=\exp [\beta(\cos \theta-1)]
$$

Both are entire analytic in $\theta$ and are of course, periodic in $\operatorname{Re} \theta$ with the period $2 \pi$. Our main results do not depend on the choices of the initial action and the recursion formula. Our main results are:

Theorem 1. If $s<2$ and $|\theta| \leqq \pi$,

$$
\left|g^{(n)}(\theta)-1\right| \leqq \operatorname{const}\left(\lambda^{s-2}\right)^{n}(\beta / 2)
$$

uniformly in $n$. Thus $g^{(n)} \rightarrow 1$ as $n \rightarrow \infty$.

Theorem 2. Let $g^{(n)}(\theta)=\tilde{g}^{(n)}(0)+\sum_{k \neq 0} \tilde{g}^{(n)}(k) e^{i k \theta}$ and let $\sum_{k \neq 0} \tilde{g}^{(n)}(k)=\delta_{n}$. Then

$$
\begin{gathered}
\tilde{g}^{(n)}(k) \geqq 0, \\
\delta_{n} \leqq(\delta)^{\lambda^{2\left(n-n_{0}\right)},}
\end{gathered}
$$

where $\delta$ is a constant less than 1 and $n_{0}>0$ is also a constant which depends on $\beta$. Ineq. (6b) holds whenever $s$ is less than 2.

Theorem 1 means that the effective actions are driven to the high temperature region by the Migdal-Kadanoff recursion formulas if $s<2$. Theorem 2, which is immediate from Theorem 1, means that the string tension is bounded from below by $(\lambda)^{-2 n_{0}}$. This corresponds to the inverse of the correlation length in the case of the statistical models.

Remark 1. (1) We can say that the Kadanoff formulas are more ferromagnetic than those of Migdal because the former have more vertical plaquettes with $\beta=\infty$ than the latter. But this difference is not seen for $D<D_{c}=4$.

(2) As is understood from our models, these recursion formulas implicitly assume the hierarchical structures in the models. In fact it is difficult to obtain the renormalization group recursion formulas in a closed form without introducing any hierarchical structure. See [7-9] for other kinds of hierarchical models.

\section{Proof of Theorem 1}

We first remark that

$$
\begin{gathered}
\left|g^{(0)}(\theta+i a)\right| \leqq g^{(0)}(\theta) \exp \left[\frac{\beta}{2} a^{2}\right], \quad \text { (Villain), } \\
\left|g^{(0)}(\theta+i a)\right| \leqq g^{(0)}(\theta) \exp \left[(1+\varepsilon) \frac{\beta}{2} a^{2}\right], \quad(\mathrm{XY}),
\end{gathered}
$$


where $a$ is any real number for the Villain action and $a \in R$ and $|a| \leqq C(C=2$ for example) in the case of the XY model type action. Inequality ( $7 \mathrm{a})$ is immediate from Eq. (4a). Since $\cos (\theta+i a)=\cos \theta+\cos \theta(\cosh a-1)-i \sin \theta \sinh a$, (7b) is also trivial. Here $\varepsilon \rightarrow 0$ as $C \rightarrow 0$.

Lemma 1. In the case of the Villain action,

$$
\left|g^{(n)}(\theta+i a)\right| \leqq g^{(n)}(\theta) \exp \left[\lambda^{-(2-s) n}\left(\frac{\beta}{2}\right) a^{2}\right]
$$

for any real $a$, and in the case of the $\mathrm{XY}$ model action,

$$
\left|g^{(n)}(\theta+i a)\right| \leqq g^{(n)}(\theta) \exp \left[(1+\varepsilon) \lambda^{-(2-s) n}\left(\frac{\beta}{2}\right) a^{2}\right]
$$

for real a such that $|a| \leqq \lambda^{2 n} C$.

Proof. We consider

M)

$$
\begin{array}{ll}
\text { M) } & g^{(1)}(\theta+i a)=(\mathscr{N})^{-1}\left[\int g^{(0)}\left(\theta+i a-\theta_{1}\right) \ldots g^{(0)}\left(\theta_{\lambda^{2}-1}\right) \prod d \theta_{i}\right]^{\lambda s}, \\
\text { K) } & g^{(1)}(\theta+i a)=(\mathscr{N})^{-1} \int\left[g^{(0)}\left(\theta+i a-\theta_{1}\right) \ldots g^{(0)}\left(\theta_{\lambda^{2}-1}\right)\right]^{\lambda s} \prod d \theta_{i} .
\end{array}
$$

Since $g^{(0)}(\theta)$ is analytic and is also periodic, we can change the contour of $d \theta_{i}$ from $-\pi \rightarrow \pi$ to $-\pi+i a_{i} \rightarrow \pi+i a_{i}$. Namely we can replace $\theta_{i}$ by $\theta_{i}+i a_{i}$. We choose $a_{i}$ $=a\left(\lambda^{2}-i\right) / \lambda^{2}$. Then $a-a_{1}=a / \lambda^{2}, a_{i}-a_{i+1}=a / \lambda^{2}$ and $a_{\lambda^{2}-1}=a / \lambda^{2}$. (This means that $|a| \leqq C \lambda^{2}$ in the case of XY model action.) Then using (7a) and (7b), we have

$$
\left|g^{(1)}(\theta+i a)\right| \leqq g^{(1)}(\theta) \times \begin{cases}\exp \left[\frac{\beta}{2} \lambda^{s} \lambda^{2}\left(a / \lambda^{2}\right)^{2}\right], & \text { Villain, } \\ \exp \left[(1+\varepsilon) \frac{\beta}{2} \lambda^{s} \lambda^{2}\left(a / \lambda^{2}\right)^{2}\right], & \mathrm{XY}\end{cases}
$$

for both of the recursion formulas of $\mathrm{M}$ and $\mathrm{K}$ type. This proves the lemma for $n=1$. The proof is completed by induction. Q.E.D.

Note that $0<g^{(n)}(\theta) \leqq 1$ for real $\theta$. This comes from the condition $g^{(n)}(\theta=0)=1$ and $g^{(n)}(0) \geqq g^{(n)}(\theta)$. The latter is immediate since $\tilde{g}^{(n)}(k) \geqq 0$, which is also explicitly shown by using $\tilde{g}^{(0)}(k) \geqq 0$. Thus if $s<2, g^{(n)}$ tends to a bounded entire function as $n \rightarrow \infty$. This is the main idea in this paper.

Lemma 2. Let $g^{(n)}(\theta)=1+a_{n} \theta^{2}+\delta g^{(n)}(\theta)$, where $|\theta| \leqq \pi$ and $\delta g^{(n)}(\theta)=O\left(\theta^{4}\right)$. Then in the case of the Villain action (taking $n$ large for the second inequality),

$$
\left|a_{n}\right| \leqq e R_{n}^{-2}, \quad\left|\delta g^{(n)}(\theta)\right| \leqq 2 e\left(\theta / R_{n}\right)^{4}
$$

for both types of the recursion formulas, where

$$
R_{n}^{2}=\lambda^{(2-s) n}\left(\frac{2}{\beta}\right)
$$

For the action of the $\mathrm{XY}$ model type, $\beta$ is replaced by $(1+\varepsilon) \beta$. 
Proof. By the Cauchy integral formula

$$
\begin{aligned}
\left|a_{n}\right| & =\left|(2 \pi)^{-1} \int g^{(n)}(z) z^{-3} d z\right| \\
& \leqq R^{-2} \max _{|z|=R}\left|g^{(n)}(z)\right| \\
& \leqq R^{-2} \exp \left[\left(\lambda^{s-2}\right)^{n}\left(\frac{\beta}{2}\right) R^{2}\right], \quad \text { (Villain) }
\end{aligned}
$$

where the contour is chosen as $|z|=R$ and Lemma 1 has been used. To minimize this upper bound, we set $R^{2}=R_{n}^{2}=\left(\lambda^{2-s}\right)^{n}(2 / \beta)$. Similarly

$$
\begin{aligned}
\left|\delta g^{(n)}\right| & =\left|\sum_{4}^{\infty}\left(\theta^{k} / 2 \pi\right) \int g^{(n)}(z) z^{-k-1} d z\right| \\
& \leqq\left(\sum_{0}^{\infty}(|\theta| / R)^{k}\right) \frac{\theta^{4}}{R^{4}} \exp \left[\left(\lambda^{s-2}\right)^{n}\left(\frac{\beta}{2}\right) R^{2}\right],
\end{aligned}
$$

and we again set $R^{2}=R_{n}^{2}$. Since $s>0, R_{n}<\lambda^{2 n} C$ for large $n$. Then these estimates still hold by replacing $\beta$ by $(1+\varepsilon) \beta$ in the case of the XY model type action. Q.E.D.

Theorem 1 obviously follows from Lemma 2.

\section{Proof of Theorem 2}

Let $g^{(n)}=\sum_{k} \tilde{g}^{(n)}(k) e^{i k \theta}$, where $\sum_{k} \tilde{g}^{(n)}(k)=1$ by the definition, and $\tilde{g}^{(n)}(k)=\tilde{g}^{(n)}(-k)$ since $g^{(n)}$ is even. Let

$$
\delta_{n}=\sum_{k \neq 0} \tilde{g}^{(n)}(k)=1-\tilde{g}^{(n)}(0) .
$$

Using $\tilde{g}^{(n)}(0)=(2 \pi)^{-1} \int g^{(n)}(\theta) d \theta$ and Lemma 2, we have

$$
0<\delta_{n} \leqq \text { const } R_{n}^{-2}, \quad\left(\text { const } \leqq \pi^{2} \text { for large } R_{n}\right)
$$

for both types of actions. Choosing $n_{0}$ sufficiently large, we assume $\delta_{n_{0}} \equiv \delta \ll 1$. We set $g^{\left(n_{0}\right)}=g$ for simplicity. They obey the following recursion formulas:

(M type)

$$
g^{(1)}(\theta)=(\mathcal{N})^{-1}\left[(1-\delta)^{\lambda^{2}}+\sum_{k \neq 0}(\tilde{g}(k))^{\lambda^{2}} e^{i k \theta}\right]^{\lambda^{s}}
$$

(K type)

$$
\tilde{g}^{(1)}(k)=(\mathcal{N})^{-1}\left[\sum_{k_{i} ; \Sigma k_{i}=k} \tilde{g}\left(k_{1}\right) \ldots \tilde{g}\left(k_{\lambda^{s}}\right)\right]^{\lambda^{2}}
$$

Since $\tilde{g}^{(0)}(k) \geqq 0$, these recursion formulas mean $\tilde{g}^{(n)}(k) \geqq 0$.

We first consider the recursion formula of $M$ type. By definition

$$
\mathscr{N}=\left[(1-\delta)^{\lambda^{2}}+\sum_{k \neq 0}(\tilde{g}(k))^{\lambda^{2}}\right]^{\lambda^{s}} \geqq(1-\delta)^{\lambda^{2+s}} .
$$


On the other hand

$$
\begin{aligned}
\delta_{1} & =\sum_{k \neq 0} \tilde{g}^{(1)}(k)=\mathscr{N}^{-1}\left\{\left[(1-\delta)^{\lambda^{2}}+\sum_{k \neq 0} \tilde{g}(k)^{\lambda^{2}} e^{i k \theta}\right]^{\lambda^{s}}-\text { const part }\right\}_{\theta=0} \\
& \leqq \mathscr{N}^{-1}\left\{\left[(1-\delta)^{\lambda^{2}}+\sum_{k \neq 0} \tilde{g}(k)^{\lambda^{2}}\right]^{\lambda^{s}}-(1-\delta)^{\lambda^{2+s}}\right\} \\
& \leqq \mathcal{N}^{-1}\left\{\left[1+\left(\sum_{k \neq 0} \tilde{g}(k)\right)^{\lambda^{2}}\right]^{\lambda^{s}}-1\right\} \\
& =\mathscr{N}^{-1}\left\{\left(1+\delta^{\lambda^{2}}\right)^{\lambda^{s}}-1\right\} \leqq(1-\delta)^{-\lambda^{2+s}}\left[\exp \left(\lambda^{s} \delta^{\lambda^{2}}\right)-1\right] .
\end{aligned}
$$

Thus choosing $\delta$ small enough,

$$
\delta_{1} \leqq 2 \lambda^{s} \delta^{\lambda^{2}} \leqq(K \delta)^{\lambda^{2}}
$$

where $K$ is a constant smaller than $2 \lambda^{s}$. This means

$$
\delta_{n} \leqq K^{\lambda^{2}+\lambda^{4}+\ldots+\lambda^{2 n}} \cdot(\delta)^{\lambda^{2 n}} \leqq\left(K^{3 / 2} \delta\right)^{\lambda^{2 n}} .
$$

Replace $n$ by $n-n_{0}$ and $K^{3 / 2} \delta$ by $\delta$ to complete the proof of Theorem 2 for the recursion formulas of the $M$ type.

In the case of $\mathrm{K}$ type, we first note $\mathscr{N} \geqq(1-\delta)^{\lambda^{2+s}}$, which follows from (14b). Moreover

$$
\begin{aligned}
\delta_{1} & =\sum_{k \neq 0} \mathscr{N}^{-1}\left\{\sum_{k_{i} ; \Sigma k_{i}=k} \tilde{g}\left(k_{1}\right) \ldots \tilde{g}\left(k_{\lambda s}\right)\right\}^{\lambda^{2}} \\
& \leqq \mathscr{N}^{-1}\left\{\sum_{k \neq 0} \sum_{k_{i} ; \Sigma k_{l}=k} \tilde{g}\left(k_{1}\right) \ldots \tilde{g}\left(k_{\lambda^{s}}\right)\right\}^{\lambda^{2}} \\
& \leqq \mathcal{N}^{-1}\left\{1-(\tilde{g}(0))^{\lambda^{s}}\right\}^{\lambda^{2}} \\
& =\mathscr{N}^{-1}\left\{1-(1-\delta)^{\lambda^{s}}\right\}^{\lambda^{2}},
\end{aligned}
$$

where we have used $\sum_{k} \tilde{g}(k)=1$ in the second line to obtain the third line. Thus we have

$$
\delta_{1} \leqq\left(K^{\prime} \delta\right)^{\lambda^{2}}
$$

as before. This completes the proof of Theorem 2 .

As an immediate corollary of this method, we have:

Theorem 3. For any dimension $D$, if $\beta$ is small enough, then $g^{(n)}(\theta) \rightarrow 1$ as $n \rightarrow \infty$. More numerically

$$
\delta_{n} \equiv \sum_{k \neq 0} \tilde{g}^{(n)}(k) \leqq(\delta)^{\lambda^{2\left(n-n_{0}\right)}},
$$

where $\delta<1$ and $n_{0}$ is a constant which depends on $\beta$.

It is almost trivial that this theorem can be extended to some non-abelian models. (In this case, $e^{i k \theta}$ are replaced by spherical harmonic functions.)

\section{String Tension and Discussions}

To calculate the string tension in the present system $(D=3)$, consider an $x_{1}-x_{2}$ plane $\Sigma$ (of size $\lambda^{N} \times \lambda^{N}$ ) in $\Xi$. Let

$$
W=\exp \left[i \sum_{b \in \partial \Sigma} \theta_{b}\right]
$$


where the sum over $\partial \Sigma$ is ordered along $\partial \Sigma$ in the usual way. In our formula, the integration over $\left\{\theta_{b} ; b \in \partial \Sigma\right\}$ is left to the final step of the recursion formulas. Thus

$$
\langle W\rangle=\frac{\int W\left(\sum \theta_{b}\right) g^{(N)}\left(\sum \theta_{b}\right) \Pi d \theta_{b}}{\int g^{(N)}\left(\sum \theta_{b}\right) \Pi d \theta_{b}}=\frac{\tilde{g}^{(N)}(1)}{\tilde{g}^{(N)}(0)},
$$

where $\tilde{g}^{(N)}(1) \leqq(K \delta)^{\lambda^{2\left(N-n_{0}\right)}}$ and $0<1-\tilde{g}^{(N)}(0) \leqq(K \delta)^{\lambda^{2\left(N-n_{0}\right)}}$.

Then the string tension $\sigma$ defined by $-\log \langle W\rangle / \lambda^{2 N}(\mathrm{~N} \gg 1)$ is bounded from below by

$$
\left(\lambda^{2 n_{0}}\right)^{-1} \log (K \delta)^{-1}
$$

Since $K \delta$ is now a fixed small constant, $n_{0}$ is defined so that

$$
\left(\lambda^{2-s}\right)^{-n_{0}}(\beta / 2) \lesssim \text { fixed small const. }
$$

Namely $n_{0}$ is chosen as the solution of

$$
(\lambda)^{-n_{0}}=\operatorname{const}(\beta)^{-\frac{1}{2-s}} .
$$

Then the string tension is bounded from below by

$$
\operatorname{const}(\beta)^{-\frac{2}{2-s}}
$$

where $s=1$ for $D=3$.

Theorem 4. For $D=3$,

$$
\sigma \geqq \operatorname{const} \beta^{-2}
$$

within the Migdal-Kadanoff recursion formulas.

Of course, we should imbed $\partial \Sigma$ in $Z^{3}$ and should take the thermodynamic limit. But the situation will not change at all since the system is now in a high temperature region by the iterations of the recursion formulas. (This check is left to the reader.) Thus we encounter a difficulty which we think serious, because we already know

$$
\text { const } e^{-c_{1} \beta} / \sqrt{\beta} \leqq \sigma \leqq \text { const } e^{-c_{2} \beta}, \quad \beta>1,
$$

where $c_{2} \cong c_{1}=(0.2527 \ldots) \pi^{2}$. See [10] for the lower bound and [11] for the upper bound. This obviously contradicts Ineq. (23). For $D=2(s=0)$, this is exactly solvable and $\sigma=-\log \tilde{g}^{(0)}(1) \cong 1 / 2 \beta$ which agrees with Ineq. $(22)\left(\tilde{g}^{(0)}(0) \equiv 1\right)$.

Thus it may be fair to say that the present formulas are too close to the two dimensional system when the dimension $D$ is less than the critical $D_{c}=4$. One may replace $\lambda^{s}=\lambda(D=3)$ in Eq. (2) or Eq. (3) by $\lambda^{2-\varepsilon}$ to strengthen the ferromagnetic interactions or to increase the effective dimensions. But this turns out to be not enough. Essentially speaking, this discrepancy comes from the fact that the present formulas lack the information about the change of the activity of the equivalent Coulomb gas system $[10,11]$ which is responsible for the factor $\exp (-c \beta)$ in the string tension. It should be mentioned, however, that the present recursion formulas exhibit the flow of the effective temperature very precisely. 
It remains to see what happens when $D=4$ and $G$ is a non-abelian compact group. In this case $\beta$ may decrease very slowly, as is predicted by the renormalization group method on the continuum space-time, and then these formulas may become rather precise. It is also interesting to see whether or not these formulas exhibit the Kosterlitz-Thouless transitions for the present abelian systems at the critical dimensions, which may be checked analytically. The work in these directions is in progress. We hope that we can report on these matters in the near future.

Acknowledgements. The author would like to thank Prof. L. Streit for kindly inviting him to the project on Mathematical Physics at $\mathrm{ZiF}$, where he enjoyed fruitful discussions with the distinguished participants. Among them, discussions with Profs. K. Gawedzki, A. Kupiainen and A. Martin were very helpful for this work.

\section{References}

1. Migdal, A.: Recursion equations in gauge field theories. JETP 69810 (1975); Migdal, A.: Phase transitions in gauge and spin-lattice systems. JETP 69, 1457 (1975)

2. Kadanoff, L.: Notes on Migdal's recursion formulas. Ann. Phys. 100, 359 (1976)

3. Collet, P., Eckmann, J.: A spin glass Model of Random couplings. Commun. Math. Phys. 93, 379 (1984);

Collet, P., Eckmann, J., Glaser, V., Martin, A.: Study of the iteration of a mapping associated to a spin glass model. Commun. Math. Phys.

4. Jose, J., Kadanoff, L., Kirkpatric, S., Nelson, S.: Renormalization, vortices, and symmetrybreaking perturbations in the two-dimensional planar model. Phys. Rev. B 16, 1217 (1977)

5. Nauenberg, M., Toussaint, D.: Migdal-Kadanoff recursion relations in SU(2) and SU(3) gauge theories. Nucl. Phys. B 190, 217 (1981)

6. Tomboulis, E.: Permanent confinement in four-dimensional non-abelian lattice gauge theory. Phys. Rev. Lett. 50, 885 (1983)

7. Dyson, F.J.: Existence of a phase-transition in a one-dimensional Ising ferromagnet. Commun. Math. Phys. 12, 91 (1969);

Dyson, F.J.: Ferromagnet with discontinuous long-range order, Commun. Math. Phys. 21, $269(1971)$

8. Gawedzki, K., Kupiainen, A.: Triviality of $\phi_{4}^{4}$ and all that in hierarchical model approximation. J. Stat. Phys. 29, 683 (1982)

9. Ito, K.R.: Trajectory of $\phi_{4}^{4}$ by block spin transformations. ZiF preprint (Bielefeld 1984)

10. Mack, G., Göpfert, M.: Proof of confinement of static quarks in 3-dimensional $U(1)$ lattice gauge theory for all values of the coupling constant. Commun. Math. Phys. 82, 545 (1982)

11. Ito, K.R.: Upper and lower bound for the string tension in the three-dimensional lattice quantum electrodynamics. Nucl. Phys. B 205, [FS 5], 440 (1982)

Communicated by G. Mack

Received March 6, 1984; in revised form April 17, 1984 
